\title{
Exploring the effects of a universal classroom management training programme on teacher and child behaviour: A group randomised controlled trial and cost analysis
}

Journal of Early Childhood Research 2017, Vol. 15(2) 174-194 (C) The Author(s) 2015

Reprints and permissions: sagepub.co.uk/journalsPermissions.nav DOI: $10.1177 / 1476718 \times 15579747$ journals.sagepub.com/home/ecr

@SAGE

\author{
Grainne Hickey', Sinead McGilloway', \\ Lynda Hyland'2, Yvonne Leckey', Paul Kelly', \\ Tracey Bywater ${ }^{3}$, Catherine Comiskey ${ }^{4}$, \\ Anne Lodge 5 , Michael Donnelly ${ }^{6}$ and \\ Donal O'Neill'
}

\begin{abstract}
Teachers frequently struggle to cope with conduct problems in the classroom. The aim of this study was to assess the effectiveness of the Incredible Years Teacher Classroom Management Training Programme for improving teacher competencies and child adjustment. The study involved a group randomised controlled trial which included 22 teachers and 217 children ( 102 boys and I I5 girls). The average age of children included in the study was 5.3 years (standard deviation $=0.89$ ). Teachers were randomly allocated to an intervention group $(n=11$ teachers; II 0 children) or a waiting-list control group $(n=11$; 107 children). The sample also included 63 'high-risk' children (33 intervention; 30 control), who scored above the cut-off $(>12)$ on the Strengths and Difficulties Questionnaire for abnormal socioemotional and behavioural difficulties. Teacher and child behaviours were assessed at baseline and 6 months later using psychometric and observational measures. Programme delivery costs were also analysed. Results showed positive changes in teachers' selfreported use of positive classroom management strategies (effect size $=0.56$ ), as well as negative classroom management strategies (effect size $=-0.43$ ). Teacher reports also highlight improvements in the classroom behaviour of the high-risk group of children, while the estimated cost of delivering the Incredible Years Teacher Classroom Management Training Programme was modest. However, analyses of teacher and child observations were largely non-significant. A need for further research exploring the effectiveness and costeffectiveness of the Incredible Years Teacher Classroom Management Training Programme is indicated.
\end{abstract}

\footnotetext{
'Maynooth University, Ireland

${ }^{2}$ Middlesex University Dubai, United Arab Emirates

3University of York, UK

${ }^{4}$ Trinity College Dublin, Ireland

${ }^{5}$ Church of Ireland College of Education, Ireland

${ }^{6}$ Queen's University Belfast, UK
}

\section{Corresponding author:}

Sinead McGilloway, Department of Psychology, Maynooth University, John Hume Building, Maynooth, Co.

Kildare, Ireland.

Email: Sinead.McGilloway@nuim.ie 


\section{Keywords}

classroom conduct, conduct problems, early intervention, prevention, socioemotional and behavioural difficulties, teacher behaviour, teacher classroom management

\section{Introduction}

Socioemotional and behavioural difficulties in an educational context are associated with academic underachievement, poor attendance and disengagement from school, as well as poorer teacher-student relationships, difficult peer relationships and low self-esteem (Evans et al., 2003; Graziano et al., 2007; Ladd, 1999). Conduct-disordered behaviour and socioemotional dysregulation during early childhood can also increase the risk of antisocial behaviour, mental health problems and economic maladjustment later in life (Bywater, 2012). Approximately 10-15 per cent of children are likely to engage in challenging behaviour, the prevalence of which has been increasing in recent years (Collishaw et al., 2004). These children need to be supported in the classroom in order to promote positive social and academic adjustment, particularly in the early years of formal education (Hyland et al., 2014).

Classroom management is one of the most important, yet challenging aspects of teaching (Coffee and Kratochwill, 2013; Reinke et al., 2011). Appropriate and effective management of the classroom environment has been shown to be crucial to child well-being and adjustment (Reddy et al., 2013; Sutherland et al., 2008). However, teachers often report struggling to cope with behavioural problems when they arise in the classroom, and many lack knowledge of research-based strategies and practices which can promote positive learning environments (Reinke et al., 2014; Webster-Stratton et al., 2008). The absence of effective classroom management can be detrimental to the development of children displaying higher levels of aggressive and disruptive behaviour in the classroom (Oliver et al., 2011). Thus, in-service classroom management training programmes are needed to help enhance teachers' classroom management skills and promote child well-being.

\section{The Incredible Years Teacher Classroom Management Programme}

The Incredible Years (IY) intervention series is described as a set of 'promising' programmes by the Blueprints for Violence Prevention and comprises separate programmes for parents, teachers and children (Webster-Stratton, 2011). The Incredible Years Teacher Classroom Management Training Programme (IYTP) is a universal intervention which aims to operationalise and transfer to teachers evidence-based strategies for classroom management. The programme is a brief, group-based intervention based on behavioural and social learning principles (Webster-Stratton, 2005a). Group discussions, role-plays and video vignettes are used to help teachers acquire teaching methods and other skills aimed at promoting positive child behaviour and decreasing challenging behaviour within the classroom. Coaching is embedded within the design of the IYTP (Reinke et al., 2012), and trained facilitators guide participants through programme delivery and assist teachers in translating processes and skills to the context of their individual classrooms (Webster-Stratton et al., 2011).

Overall, the programme aims to help teachers build positive relationships with their students and facilitate child social development and problem solving skills. The development of positive relationships in the learning environment is one of the core components of evidence-based management practices in early educational settings (Simonsen et al., 2008). Warm, supportive relationships in the classroom and positive student-teacher interactions have been found to positively 
influence child outcomes and classroom behaviour (Hamre and Pianta, 2001; Jennings and Greenberg, 2009; Lang et al., 2013; Roache and Lewis, 2011). Other proactive teaching methods promoted by the IYTP include the establishment of clear rules; setting limits and expectations for behaviour; problem solving; using fewer, more positive and clearer commands; and allowing children more time to respond to instructions. Meta-analyses of classroom management strategies have demonstrated that children demonstrate more pro-social behaviour and less disruptive behaviour in classrooms where teachers adopt a more proactive and preventative approach to classroom management (Marzano et al., 2003; Simonsen et al., 2008).

Specific skills taught during IYTP delivery include reinforcement strategies such as praise, encouragement and incentives for positive child behaviours, as well as non-coercive disciplining techniques and ignoring negative classroom behaviour in order to decrease its frequency. The use of praise, incentives and contingency strategies for behaviour modification has been widely studied and has been shown to help promote appropriate pupil behaviour and reduce disruptive behaviour (Marzano et al., 2003; Oliver et al., 2011; Raver et al., 2008). During intervention delivery, teachers are also supported by group facilitators in the development, and performance evaluation, of behaviour support plans for pupils with more challenging or conduct-disordered behaviour. Such plans consist of reinforcement strategies, as well as instructional practices and environmental supports to reduce problem behaviours, increase positive behaviours and encourage socioemotional development. Previous research has shown that the implementation of behavioural support plans can reduce disruptive behaviours and increase pro-social behaviours and compliance among at-risk children (Reinke et al., 2014).

To date, the IYTP has been evaluated largely in combination with parent- and/or child-training programmes (Webster-Stratton et al., 2004, 2008). The results of these studies show that teachers who received the IYTP training used fewer negative classroom management strategies, were more consistent when dealing with inappropriate behaviour and showed more socioemotional teaching. Improvements in child behaviour were also reported, although these effects may be due to the additional provision of parent- and/or child-training programmes.

There have been only a small number of evaluations of the IYTP as a stand-alone intervention, but most of these were based on an adapted version of the programme (Baker-Henningham et al., 2012; Raver et al., 2008; Williford and Shelton, 2008) or did not include a comparison group (Carlson et al., 2011). A non-randomised pilot evaluation of the IYTP carried out by Hutchings et al. (2007) found that teachers who received the IYTP training gave clearer, more direct commands and allowed greater opportunity for children to comply with instructions, when compared to non-trained teachers. Children in the classes of IYTP-trained teachers were also observed to be more compliant than those in the classrooms of untrained teachers. This study further highlighted teachers' positive perceptions of the programme and its impact on the classroom environment. These findings are important as classroom management training programmes must be deemed acceptable and helpful by teachers if they are to be implemented consistently and fully in the classroom.

Only two randomised controlled trial (RCT) evaluations of the non-adapted IYTP have been carried out, one in the United Kingdom (Hutchings et al., 2013) and one in the United States (Reinke et al., 2014). These studies highlight the benefits of the IYTP for teacher behaviour, particularly decreased use of negative strategies such as shouting and reprimanding students. Reinke and colleagues reported significant improvements in pro-social behaviour and reductions in disruptive behaviour, but only for a small sample of at-risk students $(n=46)$. Significant reductions in teachers' use of negative classroom management strategies were also reported by Hutchings et al. (2013). Negative child behaviours at a classroom level and among target children were also 
significantly reduced post-intervention. Despite finding no change in child pro-social behaviour, reductions in off-task behaviour in the classroom, as well as improvements in compliance among target children, were found. Thus, this trial showed benefits for both target and non-target children, as well as changes in teacher behaviour (Hutchings et al., 2013). Previous research has also provided some limited evidence for the cost-effectiveness of the IYTP, but only within the context of a multi-component intervention implemented in US settings (Foster et al., 2007; Olchowski et al., 2007).

\section{This study}

The IYTP is growing in popularity and is currently being implemented in the United States, Canada, New Zealand, Jamaica and throughout Europe including Norway, the Netherlands, Portugal, Spain, the United Kingdom and Ireland. However, there is a lack of evidence for the effectiveness of the programme, particularly in a European context, and little is known about the costs of programme implementation. This study involved a group RCT evaluation to assess the impact of the IYTP on teacher classroom management and child behaviour in Irish classrooms. We hypothesised that (1) teacher training would lead to improvements in teachers' classroom management strategies, (2) that there would be positive changes in children's behaviour in the classroom and (3) that IYTP would be more effective for 'high-risk' children (i.e. children who show high levels of conduct problems at baseline). A cost analysis was also undertaken to examine the cost of IYTP delivery.

\section{Method}

\section{Study context}

This group RCT was carried out in 11 primary schools including 8 urban schools, 2 semi-urban schools and 1 rural school in the south-west of Ireland. Seven of the schools were designated as 'disadvantaged' according to criteria from the Department of Education and Science (2005), including the proportion of pupils from local authority housing, lone parent households, large families and levels of parental unemployment. Eight schools were mixed-sex schools; one was non-denominational.

\section{Participants}

The flow of participants through the trial is shown in Figure 1. In total, 22 teachers ( 2 from each school) who taught either a Junior or Senior Infant class (the first and second year of formal primary education respectively) participated in the trial (Table 1). Only one of the teacher participants was male, although this is typical of teacher gender representation at primary school level in Ireland (Drew, 2006). In total, 445 children (average age in years $=5.4$, standard deviation $(\mathrm{SD})=0.84 ; 192$ boys and 253 girls) were taught by the teachers participating in the study, and class size ranged from 11 to 29 (average class size $=20$ children).

Approximately 12 index children from each class were included in the study. This sample was selected to yield a cross-section of child participants that was balanced in terms of 'high', 'medium' and 'low' levels of behavioural problems (see Table 2) as rated by their teachers on the teacher version of the Strengths and Difficulties Questionnaire (SDQ; Goodman, 1997). In two classrooms with fewer than 12 children, all pupils who met the inclusion criteria were included in the trial. 
Children were excluded if parental consent forms were not returned $(n=21)$ or if absent from school on two or more occasions during baseline and follow-up assessments $(n=24)$. This yielded a final sample of 217 index children (102 boys and 115 girls). The average age of index children was 5.3 years $(\mathrm{SD}=0.89)$. This sample included a total of 63 children (33 intervention and 30 control; 36 boys and 27 girls) who were identified at baseline as 'high risk' (i.e. obtained a score of 12 or more on the SDQ 'total difficulties' scale). Children below this score were categorised as 'low risk' ( $\mathrm{n}=154 ; 77$ intervention and 77 control; 88 boys and 66 girls).

\section{Procedure, randomisation and masking}

Teachers volunteered to take part in the study and provided informed consent. Families of participating children were provided with information sheets, and written informed consent was provided by all parents and guardians. Baseline assessments of teacher and child behaviour were conducted during an initial 2-month period, with post-intervention assessments carried out within the same academic year, approximately 6 months later. An independent statistician used a computer-generated random number sequence to randomly and blindly allocate teachers on a 1:1 basis to an intervention or waiting-list control group. Randomisation was conducted within schools so that one teacher from each school was allocated either to the intervention group or to a waiting-list control group.

Concealment of the allocation sequence was ensured by randomising participants after baseline assessments were completed. Participant allocation was conveyed to teachers by a member of the research team who, at post-intervention assessment, could not be blind to treatment allocation. All other researchers were blind to group allocation to minimise potential bias. Teachers were asked not to indicate to researchers whether they had received the training.

\section{Intervention}

The content of the intervention sessions is shown in Box 1. Teachers are encouraged to establish more positive relationships with pupils through the use of videotape modelling, role-plays and group discussions. They are also trained in specific skills, including developing clear classroom rules, using predictable schedules, giving praise and attention to pro-social behaviour, using encouragement and incentives, ignoring negative behaviour, using timeout and limit setting as non-aversive disciplining strategies, and facilitating child-directed play and cooperative learning opportunities. The development of behaviour support plans for specific pupils is also supported during intervention delivery.

Teacher training was delivered 1 day per month for 5 consecutive months. This monthly delivery schedule is designed to provide teachers with sufficient time to implement the newly learned management strategies, including behaviour support plans, in the classroom. During the monthlong interval between each teacher training session, teachers received one telephone call so that they could discuss the implementation of the new classroom management strategies with one of the group facilitators. Teachers also kept a written diary and provided verbal feedback to (and received feedback from) the group and the facilitators on their progress at the next session. This provided teachers and facilitators with an opportunity to identify and address any difficulties they had when implementing the new strategies and behavioural support plans in their classrooms.

Teacher substitution costs were provided to schools, and participating teachers were provided with refreshments to encourage attendance. The IYTP was delivered to teachers by two trained facilitators: one facilitator, a teacher, was fully accredited, while the other, a psychologist, was working towards accreditation during programme delivery. Group facilitators received regular 


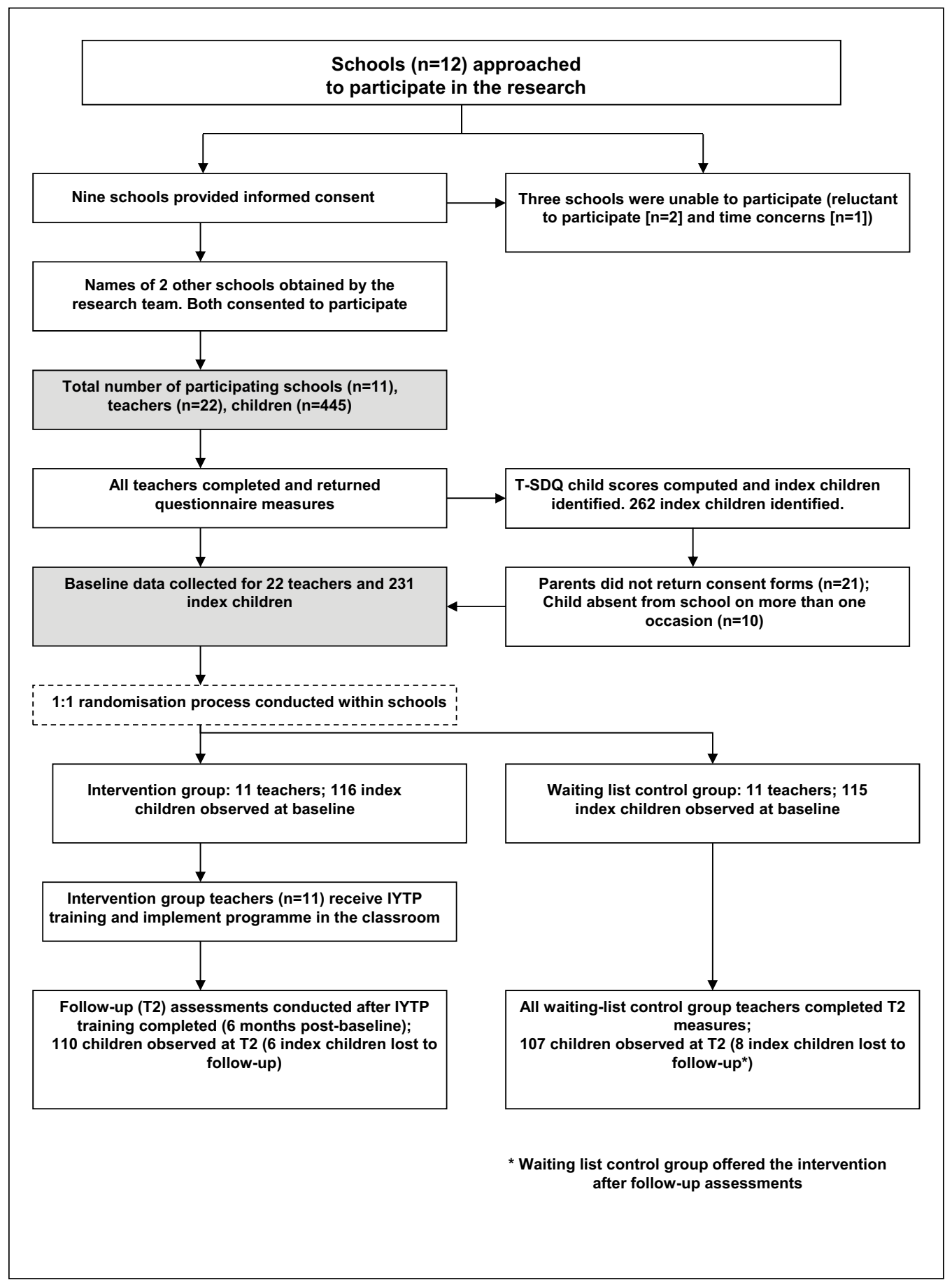

Figure I. Participant flow and group RCT design. 
Table I. Demographic information for intervention and control groups (figures are mean, standard deviation (SD) unless otherwise stated). ${ }^{\mathrm{a}}$

\begin{tabular}{lcc}
\hline Teacher characteristics & Control $(\mathrm{n}=\mathrm{II})$ & Intervention $(\mathrm{n}=\mathrm{II})$ \\
\hline $\begin{array}{l}\text { Teacher age range (years), } \mathrm{n}(\%) \\
\quad<25\end{array}$ & $\mathrm{I}(9)$ & $\mathrm{I}(9)$ \\
$25-34$ & $7(64)$ & $8(73)$ \\
$35-44$ & $\mathrm{I}(9)$ & $\mathrm{I}(9)$ \\
$45-54$ & $2(\mathrm{I})$ & $\mathrm{I}(9)$ \\
Gender & & $\mathrm{II}(\mathrm{I00})$ \\
$\quad$ Female, $\mathrm{n}(\%)$ & $10(9 \mathrm{I})$ & $8.8(7.6)$ \\
Years of teaching experience & $10.9(9.5)$ & $20(5.46)$ \\
Teacher-pupil ratio & $19(5.79)$ & Intervention $(\mathrm{n}=223)$ \\
\hline Class characteristics & Control $(\mathrm{n}=222)$ & $20.3(5.8)$ \\
\hline Class size & $20.2(5.8)$ & $5.4(0.99)$ \\
Pupil age, years & $5.4(0.77)$ & $10 \mathrm{I}(45.3)$ \\
Boys, $\mathrm{n}(\%)$ & $9 \mathrm{I}(4 \mathrm{I})$ & $\mathrm{I})$ \\
Girls, $\mathrm{n}(\%)$ & $13 \mathrm{I}(45.3)$ & Intervention $(\mathrm{n}=\mathrm{II})$ \\
\hline Index child characteristics & Control $(\mathrm{n}=107)$ & $5.4(0.9 \mathrm{I})$ \\
\hline Pupil age, years & $5.27(0.87)$ & $54(49)$ \\
Boys, $\mathrm{n}(\%)$ & $48(45)$ & $56(5 \mathrm{I})$ \\
Girls, $\mathrm{n}(\%)$ & $59(55)$ &
\end{tabular}

aNo differences between intervention and control groups using t-test and chi-square.

supervision throughout the delivery of the programme. A checklist was completed by each group facilitator after every monthly session to examine facilitator-reported treatment fidelity. The results showed that 88 per cent of all prescribed course material was covered across the five sessions and there was a high level of agreement between the group facilitators with regard to fidelity. Facilitator adherence to the intervention protocol or delivery quality was not independently validated.

Box I. Outline of the Incredible Years Teacher Classroom Management Training Programme (IYTP).

\section{Session 1}

Preventing behaviour problems: The proactive teacher

Session 2

The importance of teacher attention, coaching and praise

Session 3

Motivating children through incentives

Session 4

Decreasing inappropriate behaviour

Session 5

Teaching children to be socially competent: Emotional regulation, social skills and problem solving

Group discussions, videos and role-plays are used to support teacher skill development.

Coaching and monthly telephone calls are provided by group facilitators to support the implementation of classroom management strategies. Teachers keep a written diary of their progress.

Barriers to attendance addressed through the provision of substitution costs to schools. Participating teachers are provided refreshments during the sessions. 


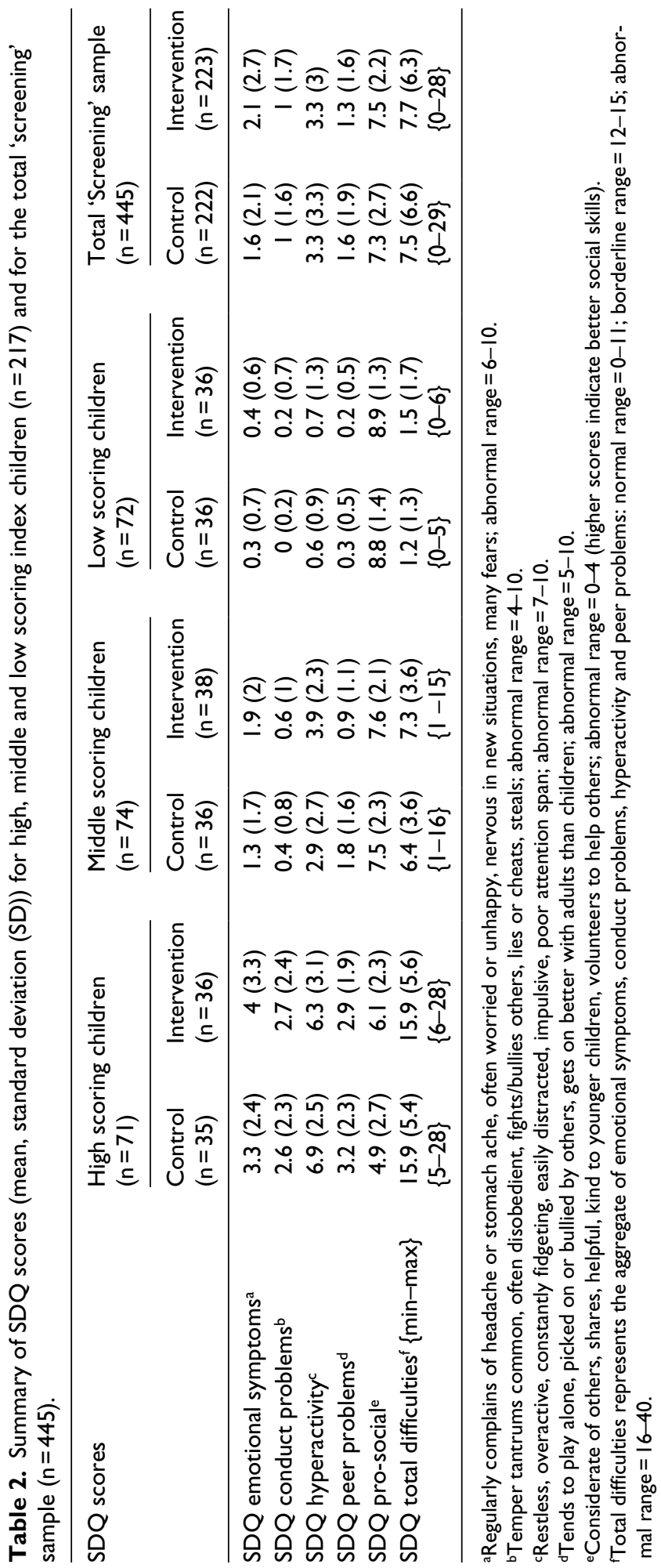




\section{Measures}

Psychometric and observational data were collected to obtain a comprehensive picture of teacher and child behaviour in the classroom. The internal consistency of scales was calculated on baseline data using Cronbach's $\alpha$.

Teacher self-report measures. The Teacher Strategies Questionnaire (TSQ; Webster-Stratton, 2005 b) was used to collect data relating to (1) self-reported frequency of teachers' use of positive and negative classroom management strategies and (2) the perceived utility of these classroom management strategies (i.e. how useful they felt these strategies are for managing behaviour in the classroom). The scale consists of 44 items $(\alpha=0.91)$. Respondents rate the extent to which statements reflect their behaviour on a 5-point Likert scale $(1=$ Rarely/Never; $5=$ Very Often).

Child behaviour measures. The teacher version of the SDQ was used to assess child problem behaviour and socioemotional well-being. The 25 -item scale consists of five subscales relating to emotional symptoms, conduct problems, hyperactivity, peer problems and pro-social behaviour. The scores on each subscale (except the pro-social scale) may be summed to generate a 'total difficulties' score $(\alpha=0.56)$. A total score of 12 or less indicates 'normal' difficulties and scores of 12-15 indicate 'borderline' difficulties, while scores of 16-40 represent 'abnormal' difficulties. A supplemental impact subscale on the SDQ was used to assess child distress and social impairment caused by socioemotional and behavioural difficulties.

Observational measure. The Teacher-Pupil Observational Tool (T-POT; Martin et al., 2010) was used to provide observations of teacher and pupil behaviours. The T-POT provides frequency counts of relevant teacher behaviours (e.g. commands, questions, warnings, praise) and child behaviours (e.g. off-task or inappropriate behaviours, peer interactions, pro-social and positive behaviours). The T-POT comprises 75 behaviour categories, including 30 teacher behaviour categories and 45 child behaviour categories. The tool allows for simultaneous coding of child behaviours, both at a classroom level and at the level of the target index child, and teacher behaviours (i.e. teacher interaction with all pupils in the classroom and direct interactions with target index children). All observations were carried out during structured lessons (i.e. reading, writing or mathematics), and each index child was observed for a total of 15 minutes.

Summary variables of observational data were created for analysis. Six observational variables of teacher behaviour were created: (1) Teacher Positives (e.g. 'Thank you', smiling, thumbs up); (2) 'Teacher Praise' (e.g. 'well done', 'nice writing'); (3) 'Teacher Negatives' (e.g. shouting or snatching an item away from a child); (4) 'Indirect Commands' (e.g. 'listen'); (5) 'Direct Commands' (e.g. 'put the book away'); and (6) 'No Opportunity' (i.e. failing to allow a child sufficient time to respond to a question or command). Four child summary observational variables were created: (1) 'Child Negatives' (e.g. shouting, screaming, making noise, hitting, sulking, snatching items away from teacher or other children); (2) 'Child Positives' (e.g. physically and verbally warm or positive behaviours); (3) 'Compliance' (e.g. responding positively to a teacher question or command); and (4) 'Non-compliance' (e.g. failing to respond, or responding negatively, to teacher instructions or questions).

Observations were carried out by fully trained coders. Reliability between coders was examined on a random selection of classroom observations (which totalled 50\% of all observations) at both baseline and post-intervention assessments. As observational variables are continuous in nature, 
inter-coder reliability on frequency counts of teacher behaviours was measured using Pearson's $r$ and showed a satisfactory level of consistency $(r=0.67)$.

\section{Analysis strategy}

Analysis of teacher behaviour. Analysis of covariance (ANCOVA) was used to examine post-intervention differences between the intervention and control groups with respect to teachers' selfreported use of classroom management strategies, taking account of treatment group, school (i.e. the school where teachers were employed) and baseline response value. ANCOVA could not be applied to the frequency counts of teacher behaviour as the assumption of independence was violated by the observational data. The analysis of the observational data on teacher behaviour, towards the classroom and towards index children, involved computing change scores (post-intervention score - baseline score) and then comparing these using independent-samples t-tests to determine whether any changes in teacher behaviour during this period differed significantly between conditions.

Analysis of child behaviour. Robust multiple linear regression was used to examine post-intervention outcomes for index children and the entire classroom, controlling for treatment group, classroom and baseline score. This approach controls for the potentially confounding effects of clustering by adjusting the standard errors; this accounts for the fact that pupils within classrooms or clusters were likely to share certain unobserved characteristics and, therefore, could not be considered independent. Sub-analyses to examine the impact of the intervention on children with differing levels of behavioural need (i.e. children who were at low and high risk of behavioural problems) were also conducted using this regression modelling approach. Effect sizes were calculated using eta squared, whereby an effect size of 0.01 denotes a small effect, 0.06 a medium effect, and 0.14 a large effect of the intervention (Cohen, 1998).

Analysis of training costs. Costs data included (1) non-recurrent initial training costs of group facilitators who delivered the IYTP, including the IYTP training fees and accommodation and travel costs of the group facilitators during the training period; (2) the pre-delivery cost of the programme such as travel and accommodation costs relating to the recruitment phase of the group RCT; (3) the group training costs (e.g. salary costs of the facilitators, group facilitator preparation, costs of providing intervention materials to teachers, room rental, and food and travel expenses for the group facilitators); and (4) substitution costs for the teachers who attended training. Costs were derived using detailed 'cost diaries' which were completed by both group facilitators.

\section{Results}

\section{Intervention attendance}

Intervention attendance was high. The mean attendance was 4.8 sessions (96\%) with nine teachers attending all 5 training sessions. Two teachers missed one session each.

\section{Baseline comparison of groups}

Teachers in the intervention group were observed to use statistically significantly more negative behaviours than their control group counterparts at baseline. There were no other 
significant differences between teachers and neither were there any significant differences between the intervention and control groups on measures of child behaviour (using independent-samples t-tests and $\left.\chi^{2}\right)$.

\section{Teacher outcomes}

The results of the ANCOVA indicated significant differences between the intervention and control groups on teachers' self-reported frequency of use and perceived usefulness of positive classroom management strategies as measured on the TSQ. Significant differences between the intervention and control groups on self-reported use of inappropriate strategies indicate that teachers in the intervention group felt that they used significantly fewer harsh and negative classroom management strategies at follow-up when compared to their control group counterparts.

The results of the independent-samples t-tests on observational data (change scores) indicated that the magnitude of change in teachers' use of negative strategies in the intervention group was significantly greater than that of the control group (Table 3). However, teachers in the intervention group were observed to use significantly more negative strategies at baseline, thereby allowing more scope for downward change. There were significant differences between intervention and control group teachers in respect of the time teachers gave to students to comply with instructions and questions ('no opportunity'), although this was due to upward change in control group teacher behaviour (teachers in the control group gave pupils less opportunity to respond to commands). There were no statistically significant differences between groups on observed use of positive classroom management strategies, praise, indirect commands and direct commands. Analysis of observed interactions between teachers and index children did not show any statistically significant intervention effects and is, therefore, not reported here.

\section{Child outcomes}

The findings from the robust regression analyses of observed child behaviour did not reveal any statistically significant effects of the intervention (Table 4). At follow-up, children in the control group were reported to show significantly more emotional symptoms (SDQ subscale) than those in the intervention group.

Outcomes for 'high-risk' children. Robust regression analyses indicated a significant difference between children in the 'high-risk' intervention and control sub-groups on the SDQ 'total difficulties' score, which favoured high-risk children in the IYTP-trained teachers' classrooms (Table 5). A statistically significant difference in peer problems (SDQ subscale) between the 'high-risk' subgroups was also found, while there was also a significant effect of the intervention with respect to the distress and social impairment experienced by 'high-risk' children in the intervention group (SDQ impact supplement scale). No significant differences were found between the intervention and control 'low risk' children across both time points.

\section{Cost analysis of the IYTP}

The recurrent and non-recurrent costs of providing the IYTP were considered within a sensitivity analysis (Table 6). Considerable travel and group facilitator overnight accommodation costs were incurred during programme delivery due to the fact that the trial was located in an area where local group facilitators were not available. These expenses, which amounted to $€ 1656.85$ ( $£ 1389.51$; US $\$ 2160.36$ ), would not apply if local group facilitators were available. When the costs relating to 







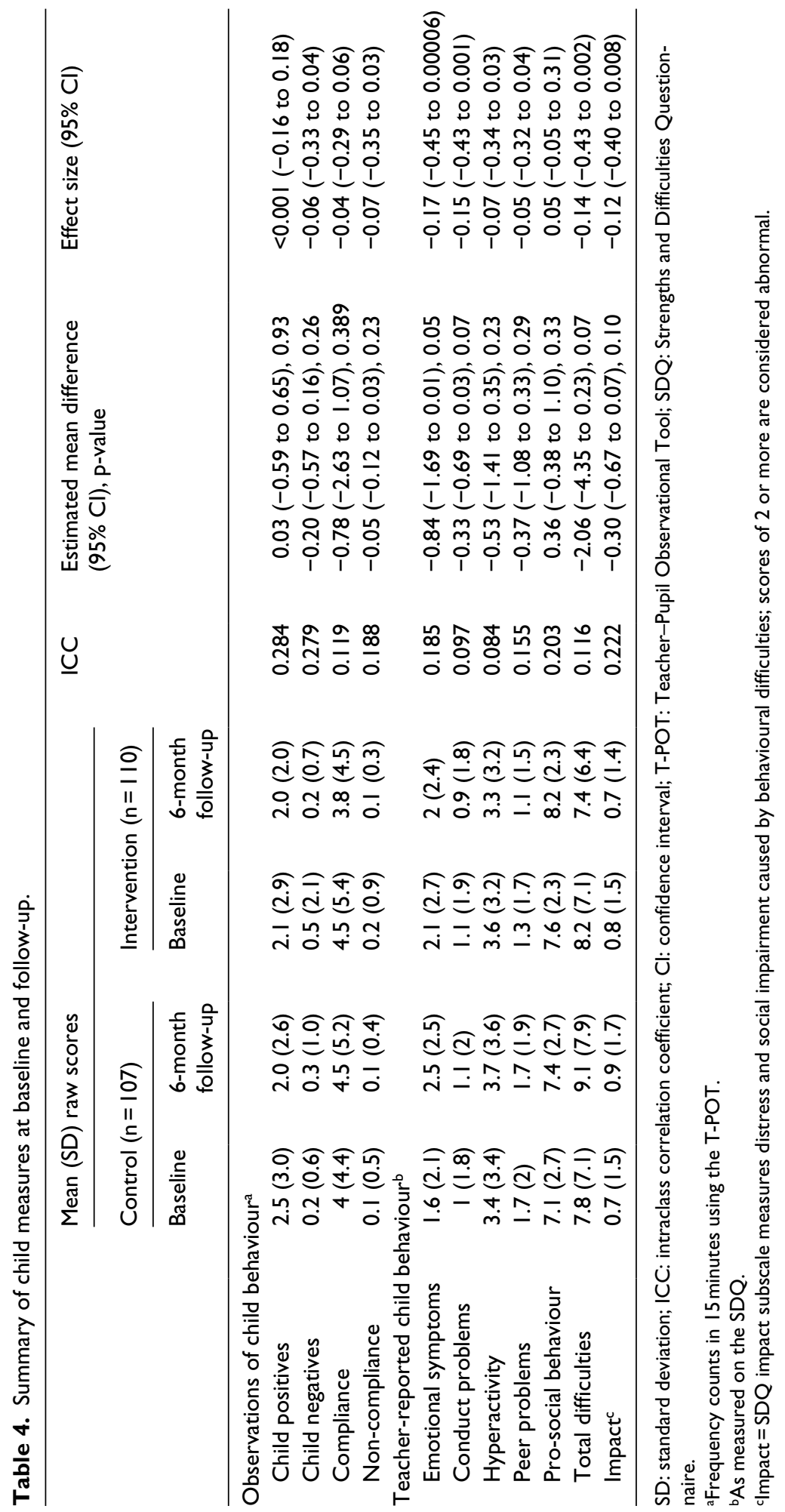




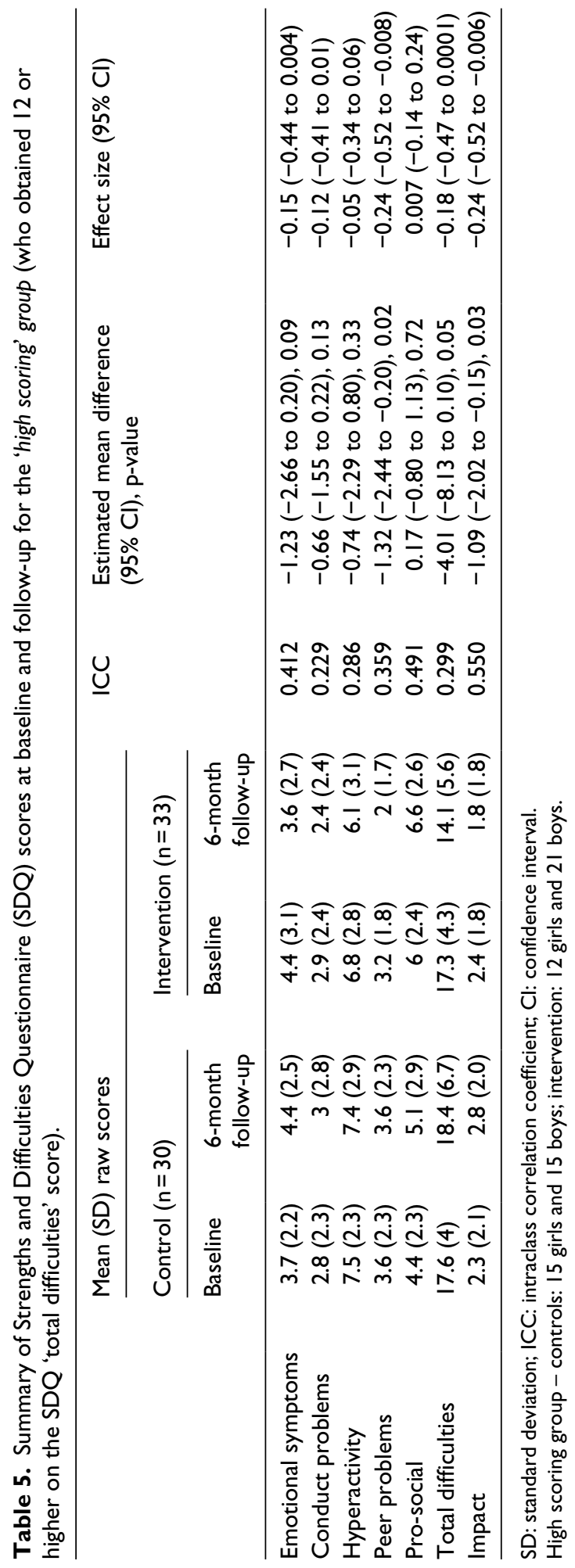


Table 6. Summary costs of the Incredible Years Teacher Classroom Management Training Programme (IYTP).

\begin{tabular}{llll}
\hline & $\begin{array}{l}\text { Total cost of } \\
\text { programme }\end{array}$ & $\begin{array}{l}\text { Average cost } \\
\text { per teacher }\end{array}$ & $\begin{array}{l}\text { Average cost } \\
\text { per client }\end{array}$ \\
\hline Non-recurrent initial training cost & $€ 2428.02$ & $€ 220.73$ & $€ 11.04$ \\
Pre-delivery cost of programme & $€ 6593.22$ & $€ 599.38$ & $€ 29.97$ \\
Group costs & $€ 5266.3$ & $€ 478.75$ & $€ 23.94$ \\
Substitution cover & $€ 7904.59$ & $€ 718.60$ & $€ 35.93$ \\
Total & $€ 22,192.13$ & $€ 2017.46$ & $€ 100.87$ \\
\hline
\end{tabular}

facilitator travel and overnight expenses were excluded, the total cost of delivering the IYTP to teachers was $€ 22,192.13$ ( $£ 18,505.48$; US $\$ 28,936.30$ ). The initial training costs of the group facilitators accounted for approximately 10 per cent of this total cost; the remainder of the cost was approximately equally divided between the remaining three cost elements which included: predelivery costs (e.g. initial preparation time such as telephone calls and contact with teachers and pre-group facilitator supervision time); group training costs (e.g. facilitator salaries, room preparation and hire, facilitator supervision, food expenses, telephone calls to participants); and substitution costs for teachers while on the training course.

The average cost per teacher was €2017.46 (£1682.31; US \$2738.50), while the cost per child (based on an average class size of 20 ) was $€ 100.87$ (£81.78; US \$131.52). These figures provide an approximation of the costs of the programme, assuming that local group facilitators are used in the delivery. When the travel and accommodation costs of the two group facilitators were factored into the cost analysis, the total cost of delivering the IYTP increased marginally to $€ 23,848.98$ ( $£ 20,004.37$; US $\$ 32,654.43)$, as did the average costs per teacher ( $€ 2168.08 ; £ 1818.57$; US $\$ 2968.67)$ and per child (€108.40; £90.92; US\$116.51).

\section{Discussion}

This study is one of a small number of studies that have explored the effectiveness of the standalone, unadapted IYTP on teacher classroom management and child classroom behaviour; uniquely, it also explored the costs of delivering the programme on a stand-alone basis. The findings from this study in relation to teacher behaviour are somewhat more mixed than previous evaluations of the IYTP (e.g. Reinke et al., 2014; Webster-Stratton et al., 2008). Analyses of observed teacher behaviour were largely non-significant. While a significant reduction in teachers' use of negative or coercive strategies was found, at baseline teachers in the intervention group used more negative strategies than their control group counterparts and they also remained higher in their observed use of negative strategies post-intervention than the control group at baseline. Teacher self-report data, which demonstrated significant intervention effects on teachers' attitudes towards - and selfreported use of - positive and proactive strategies, support the overall utility of the programme. It is possible that these findings may reflect, at least in part, teachers' attitudes towards the intervention (due to awareness that the intervention was aimed at changing teacher behaviour).

No significant differences were found in this study on observed child behaviour or child prosocial behaviour. There were teacher-reported reductions in disruptive and negative child classroom behaviour among the 'high-risk' group, as well as the alleviation of negative emotional symptoms. These findings should be interpreted with caution as changes may reflect teacher perceptions rather than actual change in child behaviour. However, previous research has shown that 
classroom management training programmes help to improve the classroom interaction between teachers and children who display more disruptive behaviour (Leflot et al., 2010). It may be possible that teacher interactions with the smaller subset of 'high-risk' children were more positive post-intervention, when compared to other children in the class, and the observational tool used in this study may not have been sufficiently sensitive to pick up such changes in teacher behaviour. Moreover, the finding that high-risk children benefit more from classroom management interventions is consistent with previous studies (Hutchings et al., 2013; Kellam et al., 1998).

Research has also found that improving the behaviour of high-risk children and reducing the levels of peer rejection more generally within the classroom can help to create a nurturing social and developmental context that may be particularly beneficial for at-risk children. Positive schooling experiences can provide an important buffer in the lives of at-risk children and may help to attenuate the adverse effects of early childhood maladjustment in the longer term (Lang et al., 2013; Van Lier et al., 2005). Indeed, recent research (Kellam et al., 2014) demonstrated that effective classroom management interventions, implemented in the early years, can reduce the risk of risky sexual behaviours and drug abuse later in life.

It is notable that the results of this study are more mixed than previous evaluations. It is difficult to know why this may be the case without further more large-scale research, but there may be a number of possible reasons for these findings. First, other IYTP studies have evaluated adapted or combined programmes which incorporated parent and/or child social skills training (e.g. BakerHenningham et al., 2012; Webster-Stratton et al., 2008) and which may have contributed to enhanced child outcomes. Second, class size in this study was somewhat larger than in research conducted in the United Kingdom (e.g. Hutchings et al., 2013). Indeed, class size can negatively impact the levels of, and challenges in managing, socioemotional and behavioural difficulties (Hyland et al., 2014). Third, previous studies have focussed on at-risk children (Reinke et al., 2014), but the majority of children in this study were within the normal range for child behaviour problems, while frequency counts at baseline of observed child negatives and non-compliance were low. Finally, cultural differences between the Irish educational system and those elsewhere may also have impacted the study outcomes. For example, teaching methods in Irish classrooms have been found to be more formal, while teachers also tend to engage in more didactic, 'whole class' teaching than in other countries (Department of Education and Science (DES), 2004). Thus, the manner in which classroom management strategies were implemented by Irish teachers may differ somewhat from those elsewhere, although this requires further investigation.

The results of the cost analysis suggest that the costs of the IYTP are modest. The average cost of providing IYTP training per child is very small $(<2 \%)$ when compared to the annual expenditure on education in Ireland (€7172; £6016; US \$9823). Irish expenditure on primary education is greater than the European Union average (€6459; £5418; US \$8838) and comparable to that of the UK (€7942; £6646; US \$10,867) and the OECD average (€6903; £5776; US \$9446) (Eurostat, 2012; OECD, 2013). On average, 94 per cent of primary education expenditure across all OECD countries is directed towards core educational services (e.g. teachers, school buildings, books, educational materials and school administration). Thus, the costs of implementing the IYTP in Ireland may be comparable to elsewhere. While differences in primary educational funding across different geographical contexts may, to some extent, limit the generalisability of the findings here, very little cost information currently exists on this (or any other similar) programme elsewhere in the world.

\section{Study strengths and limitations}

This study is one of only a very small number which have been undertaken internationally to assess the effectiveness of the unadapted IYTP and is the first to examine the costs of the programme as 
a stand-alone intervention. Psychometric and observational methodologies were combined to provide a comprehensive assessment of the impact of the IYTP on teacher and child classroom behaviour. All observations were carried out by trained researchers, while the intervention was delivered by fully certified facilitators. Strict randomisation procedures were applied and a comprehensive error audit was conducted to ensure high-quality data. An excellent level of intervention attendance by teachers was also reported. The research retention rate was also high with all 22 teachers and 94 per cent of index children retained in the study at follow-up.

The study also has a number of limitations. Due to practical constraints, one observer was not blind to teacher and classroom group allocation for post-intervention follow-up assessments. However, the remaining observers were blinded and stringent observation reliability checks were carried out on half of all classroom observations to ensure that any potential observer bias did not affect the quality of the data. The sample size for this exploratory trial was small and, therefore, the power of the study to detect changes in child behaviour was low. Randomisation in this study was restricted by school to ensure that the intervention and control groups were comparable; however, this may have given rise to some 'contamination effects' if the intervention group teachers had shared their newly learnt skills with their control group colleagues. However, the general pattern in the teacher behaviour data of the observed control group suggests that, if any contamination did occur, its effects were minimal.

Follow-up data were collected immediately after the intervention ended (i.e. before the school year ended) which may not have allowed sufficient time for the teachers to implement the full range of skills acquired through training. It is possible, therefore, that only initial improvements in teacher classroom management and child conduct were observed. Finally, although observations were used to assess teacher behaviour, direct teacher interactions with, and behaviour towards, high-risk children were not observed. In addition, teachers were not blind to intervention; thus, teacher reports of changes in the socioemotional and behavioural well-being of at-risk children in their classrooms must be interpreted with caution.

\section{Conclusion}

Ineffective classroom management can undermine the socioemotional and behavioural development of at-risk children (Oliver et al., 2011). Class sizes are increasing and the prevalence of conduct problems is escalating. Teachers also frequently report feelings of inadequacy when managing complex classroom environments (Hyland et al., 2014). Importantly, proactive teaching strategies and positive teacher-pupil relationships play an important role in the personal development and psychological adjustment of young children, particularly those who are at risk of conduct disorder and poor academic and social outcomes (Webster-Stratton et al., 2011). Teacher training programmes are recommended for targeting child classroom behaviour, and such interventions are likely to reach many more children than parent-training (Olchowski et al., 2007). This highlights the importance of providing appropriate professional development and in-service education opportunities for teachers that focus on managing the classroom environment and tackling conduct problems in the early years, particularly for those who continue to use negative and coercive teaching strategies (Roache and Lewis, 2011).

The IYTP provides training in a number of evidence-based classroom management strategies and practices including proactive teaching methods, using reinforcement schedules and building positive relationships within the classroom environment (Marzano et al., 2003; Simonsen et al., 2008). Our findings demonstrate that the programme is inexpensive to implement; however, the findings relating to teacher behaviour and child outcomes are mixed. Teacher reports support the positive effect that teacher training can have on teacher skills and the classroom behaviour of 
high-risk children. However, observations of the classroom did not corroborate teacher report, despite some trends in the expected direction.

This study is only the second to be carried out in a European context to explore the effectiveness of the IYTP; however, the programme is currently being implemented and evaluated in several other countries in Europe such as the United Kingdom, Portugal, the Netherlands and Norway, as well as Canada, New Zealand and the United States (Hutchings, 2012). Furthermore, a large-scale RCT evaluation and cost-effectiveness study of the IYTP is currently underway in the United Kingdom (Ford et al., 2012). This type of further research is important in promoting a greater understanding of the full impact of the programme on teacher and child outcomes, as well as its adaptability and applicability to different cultural contexts, all of which are important if the programme is to be rolled out on a large-scale basis.

\section{Future research}

The preventative impact of interventions can be difficult to detect, and longer follow-up time frames are recommended in order to better document such outcomes (Weisz et al., 2005). Patterns in the data point towards a small deterioration in the classroom behaviour of control group children. In the absence of any intervention, it is possible that continuing conduct problems may reinforce a maladaptive form of behavioural engagement more generally within the class, thereby leading to an escalation of inappropriate classroom behaviour and more entrenched conduct problems in the longer term (Kellam et al., 1998). Thus, further research is needed to evaluate the longterm effectiveness and implementation of the IYTP.

Economic evaluations of these types of programmes are rare, and yet these are a crucial consideration for policy makers and schools (Furlong et al., 2012). Exploration of the costeffectiveness of the IYTP, and classroom management programmes more generally, is needed. Furthermore, if programmes such as the IYTP are to be implemented more widely, several factors must be considered, including the sustainability of optimal teacher practices over time, the availability of appropriately trained facilitators, the ongoing monitoring of intervention delivery and the need to develop effective partnerships between educational practice and mental health services to ensure that appropriate, effective and timely support is made available for both educational professionals and vulnerable children alike. Future research should also explore factors that contribute to long-run implementation of, and fidelity to, classroom management programmes. Understanding the mechanisms and processes which are important to successful programme implementation and efficacy can contribute to the development of improved interventions which can, in turn, enable educators to achieve optimal outcomes for pupils in their care.

\section{Acknowledgements}

We extend a sincere thanks to Archways for their support and facilitation of this research and to all of the schools who participated in the study. We also thank all of the teachers and parents for their cooperation throughout the research process, as well as the group facilitators.

\section{Declaration of conflicting interests}

The authors declare that there is no conflict of interest. 


\section{Funding}

This research was funded by the Atlantic Philanthropies, with additional support from the Dormant Accounts Fund.

\section{References}

Baker-Henningham H, Scott S, Jones K, et al. (2012) Reducing child conduct problems and promoting social skills in a middle-income country: cluster randomised controlled trial. British Journal of Psychiatry 201(2): 101-108.

Bywater T (2012) Perspectives on the Incredible Years programme: psychological management of conduct disorder. British Medical Journal 201(2): 85-87.

Carlson J, Tiret H, Bender S, et al. (2011) The influence of group training in the Incredible Years Teacher Classroom Management programme on preschool teachers' classroom management strategies. Journal of Applied School Psychology 27(2): 134-154.

Coffee G and Kratochwill TR (2013) Examining teacher use of praise taught during behavioural consultation: implementation and generalization considerations. Journal of Educational and Psychological Consultation 23(1): 1-35.

Cohen J (1988) Statistical Power Analysis for the Behavioural Sciences. Hillsdale, NJ: Lawrence Erlbaum Associates.

Collishaw S, Maughan B, Goodman R, et al. (2004) Time trends in adolescent mental health. Journal of Child Psychology and Psychiatry 45(8): 1350-1362.

Department of Education and Science (2004). OECD Thematic Review of Early Childhood Education and Care Policy in Ireland. Dublin: Department of Education and Science.

Department of Education and Science (2005) Delivering equality of opportunity in schools: an action plan for educational and social inclusion. Stationary Office. Available at: https://www.education.ie/en/ Publications/Policy-Reports/Delivering-Equality-Of-Opportunity-In-Schools-An-Action-Plan-forEducational-Inclusion-Summary.pdf

Drew E (2006) Facing Extinction? Why Men Are Not Attracted to Primary Teaching. Dublin: The Liffey Press.

Eurostat (2012) Key figures on Europe 2012. Available at: http://epp.eurostat.ec.europa.eu/cache/ITY OFFPUB/KS-EI-12-001/EN/KS-EI-12-001-EN.PDF (accessed 16 November 2013).

Evans J, Harden A, Thomas J, et al. (2003) Support for pupils with Emotional and Behavioural Difficulties (EBD) in mainstream primary classrooms: a systematic review of the effectiveness of interventions. Report, Research Evidence in Education Library. EPPI-Centre, Social Science Research Unit, Institute of Education, London.

Ford T, Edwards V, Sharkey D, et al. (2012) Supporting teachers and children in schools: the effectiveness and cost-effectiveness of the Incredible Years teacher classroom management programme in primary school children: a cluster randomised controlled trial, with parallel economic and process evaluations. BMC Public Health 12: 719-735.

Foster EM, Olchowski AE and Webster-Stratton C (2007) Is stacking intervention components cost-effective? An analysis of the Incredible Years programme. Journal of the American Academy of Child and Adolescent Psychiatry 46(11): 1414-1424.

Furlong M, McGilloway S, Bywater T, et al. (2012) Behavioural/cognitive-behavioural group-based parenting interventions for children age 3-12 with early onset conduct problems. Campbell Systematic Reviews 2: CD008225.

Goodman R (1997) The Strengths and Difficulties Questionnaire: a research note. Journal of Child Psychology and Psychiatry 38(5): 581-586.

Graziano PA, Reavis RD, Keane SP, et al. (2007) The role of emotion regulation and children's early academic success. Journal of School Psychology 45(1): 3-19.

Hamre BK and Pianta RC (2001) Early teacher-child relationships and the trajectory of children's school outcomes through eighth grade. Child Development 72(2): 625-638.

Hutchings J (2012) Support for teachers around the world. Better: Evidence-Based Education 5(1): 18-19. 
Hutchings J, Daley D, Jones K, et al. (2007) Early results from developing and researching the WebsterStratton Incredible Years Teacher Classroom Management Training Programme in North West Wales. Journal of Children's Services 2(3): 15-26.

Hutchings J, Martin P, Daley D, et al. (2013) The effects of a teacher classroom management programme on the classroom behaviour of children with and without behaviour difficulties. Journal of School Psychology 51(5): 571-585.

Hyland L, Ni Mhaille G, McGilloway S, et al. (2014) Conduct problems in young, school-going children in Ireland: prevalence and teacher response. School Psychology International 35(5): 516-529.

Jennings P and Greenberg M (2009) The prosocial classroom: teacher social and emotional competence in relation to student and classroom outcomes. Review of Educational Research 79(1): 491-525.

Kellam SG, Ling X, Rolande M, et al. (1998) The effect of level of aggression in the first grade classroom on the course and malleability of aggressive behavior into middle school. Development and Psychopathology 12(1): 165-185.

Kellam SG, Wang W, Mackenzie ACL, et al. (2014) The impact of the good behavior game, a universal classroom-based preventive intervention in first and second grades, on high-risk sexual behaviors and drug abuse and dependence disorders into young adulthood. Prevention Science 15(Suppl. 1): 6-18.

Ladd GW (1999) Peer relationships and social competence during early and middle childhood. Annual Review of Psychology 50(1): 333-359.

Lang I, Marlow R, Goodman R, et al. (2013) Influence of problematic child-teacher relationships on future psychiatric order: population survey with 3-year follow-up. British Journal of Psychiatry 202(5): 336341.

Leflot G, Van Lier PAC, Onghena P, et al. (2010) The role of teacher behavior management in the development of disruptive behaviors: an intervention study with the good behavior game. Journal of Abnormal Child Psychology 38(6): 869-882.

Martin PA, Daley D, Hutchings J, et al. (2010) The Teacher-Pupil Observation Tool (T-POT): development and testing of a new classroom observation measure. School Psychology International 31(3): 229-249.

Marzano RJ, Marzano JS and Pickering DJ (2003) Classroom Management That Works: Research-Based Strategies for Every Teacher. Alexandria, VA: Association for Supervision and Curriculum Development.

Organisation for Economic Co-operation and Development (OECD) (2013) Education at a Glance 2013: OECD Indicators. Paris: OECD Publishing. DOI: 10.1787/eag-2013-en

Olchowski AE, Foster EM and Webster-Stratton C (2007) Implementing behavioural intervention components in a cost-effective manner: analysis of the Incredible Years program. Journal of Early Intensive Behavioural Intervention 4(1): 284-304.

Oliver R, Wehby J, Daniel J (2011) Teacher classroom management practices: effects on disruptive or aggressive student behavior. Campbell Systematic Reviews 7(4). DOI: 10.4073/csr.2011.4

Raver CC, Jones SM, Li-Grining CP, et al. (2008) Improving preschool classroom processes: preliminary findings from a randomized trial implemented in Head Start settings. Early Childhood Research Quarterly 63(3): 235-255.

Reddy LA, Fabiano G, Dudek CM, et al. (2013) Instructional and behavioral management practices implemented by elementary general education teachers. Journal of School Psychology 51(6): 683-700.

Reinke WM, Stormont M, Herman KC, et al. (2011) Supporting children's mental health in schools: teacher perceptions of needs, roles, and barriers. School Psychology Quarterly 26(1): 1-13.

Reinke WM, Stormont M, Herman KC, et al. (2014) Use of coaching and behaviour support planning for students with disruptive behaviour within a universal classroom management program. Journal of Emotional and Behavioral Disorders 22(2): 74-82.

Reinke WM, Stormont M, Webster-Stratton C, et al. (2012) The Incredible Years Teacher Classroom Management Programme: using coaching to support generalisation to real-world classroom settings. Psychology in the Schools 49(5): 416-428.

Roache JE and Lewis R (2011) The carrot, the stick, or the relationship: what are the effective disciplinary strategies? European Journal of Teacher Education 34(2): 233-248.

Simonsen B, Fairbanks S, Briesch A, et al. (2008) Evidence-based practices in classroom management: considerations for research to practice. Education and Treatment of Children 31(3): 351-380. 
Sutherland K, Lewis-Palmer T, Stichter JP, et al. (2008) Examining the influence of teacher behavior and classroom context on the behavioral and academic outcomes for students with learning and behavior problems. Journal of Special Education 41(4): 209-222.

Van Lier PAC, Vuijk PJ and Crijnen AAM (2005) Understanding mechanisms of change in the development of antisocial behaviour: the impact of a universal intervention. Journal of Abnormal Child Psychology 33(5): 521-535.

Webster-Stratton C (2005a) The Incredible Years parents, teachers, and children training series: early intervention and prevention programs for young children. In: Jensen PS and Hibbs ED (Eds) Psychosocial Treatments for Child and Adolescent Disorders. Washington, DC: American Psychological Association, pp. 435-474.

Webster-Stratton C (2005b) Teacher Classroom Management Strategies Questionnaire. Available at: http:// incredibleyears.com/download/resources/teacher-pgrm/American\%20Teacher\%20Strategies\%20 Questionnaire.pdf (accessed 16 November 2013).

Webster-Stratton C (2011) The Incredible Years: Parents, Teachers and Children's Training Series. Seattle, WA: The Incredible Years, Inc.

Webster-Stratton C, Reid JM and Hammond M (2004) Treating children with early-onset conduct problems: intervention outcomes for parent, child and teacher training. Journal of Clinical Child and Adolescent Psychology 33(1): 105-124.

Webster-Stratton C, Reid JM and Stoolmiller M (2008) Preventing conduct problems and improving school readiness: evaluation of the Incredible Years teacher and child training programs in high-risk schools. Journal of Child Psychology and Psychiatry 49(5): 471-488.

Webster-Stratton C, Reinke WM, Herman KC, et al. (2011) The Incredible Years Teacher Classroom Management Training: the methods and principles that support fidelity of training Delivery. School Psychology Review 40(4): 509-529.

Weisz JR, Sandler IN, Durlak JA, et al. (2005) Promoting and protecting youth mental health through evidence-based prevention and treatment. American Psychologist 60(6): 628-648.

Williford AP and Shelton TL (2008) Using mental health consultation to decrease disruptive behaviors in preschoolers: adapting an empirically-supported intervention. Journal of Child Psychology, Psychiatry and Allied Disciplines 49(2): 191-200. 